\title{
Biochemistry, proteomics, and phosphoproteomics of plant mitochondria from non-photosynthetic cells
}

\author{
Jesper F. Havelund ${ }^{1}$, Jay J. Thelen ${ }^{2}$ and lan M. Møller ${ }^{1 *}$ \\ ${ }^{1}$ Department of Molecular Biology and Genetics, Science and Technology, Aarhus University, Slagelse, Denmark \\ ${ }^{2}$ Department of Biochemistry and Interdisciplinary Plant Group, University of Missouri-Columbia, Columbia, MO, USA
}

\author{
Edited by: \\ Nicolas L. Taylor, The University of \\ Western Australia, Australia \\ Reviewed by: \\ Chun Pong Lee, University of \\ Oxford, UK \\ Carmela Giglione, Centre National \\ de la Recherche Scientifique, France \\ Allan Rasmusson, Lund University, \\ Sweden

\section{*Correspondence:} \\ lan M. Møller, Department of \\ Molecular Biology and Genetics, \\ Science and Technology, Aarhus \\ University, Forsøgsvej 1, \\ DK-4200 Slagelse, Denmark. \\ e-mail: ian.max.moller@agrsci.dk
}

\begin{abstract}
Mitochondria fulfill some basic roles in all plant cells. They supply the cell with energy in the form of ATP and reducing equivalents $[N A D(P) H]$ and they provide the cell with intermediates for a range of biosynthetic pathways. In addition to this, mitochondria contribute to a number of specialized functions depending on the tissue and cell type, as well as environmental conditions. We will here review the biochemistry and proteomics of mitochondria from non-green cells and organs, which differ from those of photosynthetic organs in a number of respects. We will briefly cover purification of mitochondria and general biochemical properties such as oxidative phosphorylation. We will then mention a few adaptive properties in response to water stress, seed maturation and germination, and the ability to function under hypoxic conditions. The discussion will mainly focus on Arabidopsis cell cultures, etiolated germinating rice seedlings and potato tubers as model plants. It will cover the general proteome as well as the posttranslational modification protein phosphorylation. To date 64 phosphorylated mitochondrial proteins with a total of 103 phosphorylation sites have been identified.
\end{abstract}

Keywords: plant mitochondria, proteomics, mitochondrial isolation, protein phosphorylation

\section{INTRODUCTION}

All living plant cells contain mitochondria, the organelle where organic molecules are oxidized to produce energy, in the form of ATP and reducing equivalents, as well as metabolic intermediates for use in biosynthetic processes. However, mitochondria have a number of other important functions depending on the cell and tissue type, as well as environmental conditions. In theory, each of these factors affects the properties of the mitochondria and the composition of its proteome. While this has been demonstrated at the biophysical level (Douce and Neuburger, 1989) and for some enzymes and complexes, we are entering an era where comprehensive protein profiling is possible to quantitatively assess mitochondrial remodeling in response to genetic and environmental cues. In anticipation and preparation for this époque we review critical aspects of the field including: (1) methods for isolation of ultra-pure mitochondria (for proteome interrogation); (2) properties of mitochondria (to characterize function); and (3) the current status of mitochondrial proteome investigations. Due to space constraints, we focus this review on mitochondria from non-photosynthetic tissues, which can be considered the "reference state" for comparative studies. These mitochondria perform the same basic functions and would therefore be expected to contain many of the same proteins as mitochondria from photosynthetic tissues.

\section{PURIFICATION OF MITOCHONDRIA FROM NON-GREEN TISSUES}

As is the case for all studies of organellar proteomics, a mitochondrial isolation procedure must remove all contaminants and give a clean preparation containing only mitochondria. Biochemical studies of mitochondrial metabolism often require severalmg protein. Therefore, access to large amounts of starting material has been an important factor when selecting the species and tissue to study.

During the 1950-1970's, non-photosynthetic tissues such as etiolated seedlings or storage tissues were usually employed for the isolation of mitochondria by differential centrifugation probably because a major, visible contaminant-thylakoid membranes - was absent. Actually, crude mitochondria from storage tissues are generally contaminated by amyloplast membranes (Neuburger et al., 1982), but they do not interfere with measurements of oxygen consumption, a standard method in the study of mitochondrial metabolism. When rate-zonal, density gradient purification of plant mitochondria was introduced, it was initially applied to potato tubers. Step sucrose gradients were first introduced (Douce et al., 1972) and later Percoll gradients (Neuburger et al., 1982; Struglics et al., 1993; Considine et al., 2003), which had the great advantage over sucrose gradients that the mitochondria were not exposed to large changes in osmolarity. Not only does Percoll gradient centrifugation remove major contaminants like plastids and peroxisomes, it also removes mitochondria with damaged outer and/or inner membranes (Neuburger et al., 1982; Struglics et al., 1993).

Using Percoll gradient centrifugation, purified mitochondria have now been isolated from a range of non-green tissues from a number of species (Moreau and Romani, 1982; Liden and Møller, 1988; Fredlund et al., 1991; Lind et al., 1991; Millar et al., 2001; Bardel et al., 2002; Robson and Vanlerberghe, 2002; Qin et al., 
2009; Lee et al., 2011). This has usually given a very significant improvement in mitochondrial purity and intactness and a concomitant increase in rates of respiration, which indicates that the crude mitochondria previously used had sometimes contained less than $50 \%$ undamaged mitochondria, on a protein basis (e.g., Moreau and Romani, 1982). In the case of purified potato tuber mitochondria, contamination by plastid envelope and peroxisomes could be calculated to be $<0.5 \%$ on a protein basis (Neuburger et al., 1982; Struglics et al., 1993). Percoll-purified mitochondria from Arabidopsis cell cultures, roots and shoots can be further purified by free-flow electrophoresis (Eubel et al., 2007; Lee et al., 2011). For cell culture mitochondria, this method decreases the contamination by peroxisomal and plastidic proteins by an estimated 5- to 10-fold (Eubel et al., 2007).

\section{BIOCHEMICAL PROPERTIES OF MITOCHONDRIA FROM NON-GREEN TISSUES BASIC PROPERTIES}

Mitochondria are semi-autonomous, membrane-bound organelles. Consistent with their endosymbiotic origin, plant mitochondria contain small circular genomes. Varying with species, 30-40 proteins are encoded in the mitochondrial DNA, transcribed and translated in the matrix on bacterial-like ribosomes (Kubo and Newton, 2008). The remaining proteins making up the mitochondrial proteome are imported from the cytosol mainly through two large protein complexes, TIM and TOM (Translocator Inner/Outer Membrane) (Lister et al., 2005).

Isolated mitochondria from non-green cells and tissues oxidize a variety of substrates, including most of the tricarboxylic acid (TCA) cycle intermediates, with good coupling indicating that the TCA cycle is fully functional and that the four electron transport chain (ETC) complexes are present together with ATP synthase (Douce and Neuburger, 1989). In addition, the ETC of mitochondria from non-green tissues contain up to four alternative $\mathrm{NAD}(\mathrm{P}) \mathrm{H}$ dehydrogenases (DH) (Rasmusson et al., 2004), the alternative oxidase (Vanlerberghe and McIntosh, 1997), and has the uncoupling protein associated with it (Vercesi et al., 1995; Zhu et al., 2011). A range of DHs in the matrix feed electrons into the ETC at ubiquinone (UQ) - proline DH, lactate $\mathrm{DH}$, and DHs involved in branched amino acid degradation via electron-transfer flavoprotein and electron-transfer flavoprotein: quinone oxidoreductase. On the outer surface of the inner membrane glycerol-3-phosphate DH also feeds electrons into the UQ pool, while L-galactono-1,4-lactone $\mathrm{DH}$, the last enzyme in ascorbate biosynthesis, feed electrons into cytochrome c. The presence and amount of these enzymes in the mitochondria depends on species, tissue and environmental conditions (reviewed by Rasmusson et al., 2008; Rasmusson and Møller, 2011). To interact with other subcellular compartments within the cell, the inner mitochondrial membrane contains a number of transport proteins for the exchange of metabolites, coenzymes, etc. (Laloi, 1999; Palmieri et al., 2009).

Isolated mitochondria from green tissues do not differ much in the basic properties of the ETC and the TCA cycle. However, "green" mitochondria differ in the balance between the different substrates used. This is especially true for glycine, the product of photorespiration, which is oxidized at high rates by "green" mitochondria, but also their amino acid metabolism differs (e.g., Lee et al., 2008).

\section{RESPONSES TO HYPOXIA, DROUGHT, AND DESICCATION}

Plants can be exposed to hypoxic conditions as a result of flooding (Greenway et al., 2006). However, even under normoxic external conditions, the central parts of dense, metabolically active and/or bulky non-photosynthetic tissues can experience hypoxic conditions because the diffusion of oxygen into the tissue cannot keep up with the rate of removal by respiration (Geigenberger, 2003). This leads to metabolic changes such as the induction of ROSdegrading enzymes, presumably to prevent post-anoxic injury (Geigenberger, 2003), and higher activity of the enigmatic formate dehydrogenase (FDH) (Bykova et al., 2003b), one of the most abundant proteins in potato tuber mitochondria (Colas Des Francs-Small et al., 1993). The function of FDH may be to remove formate, but the source of formate is unknown and the reason the enzyme is so abundant is obscure (Igamberdiev, 1999; Ambard-Bretteville et al., 2003).

Drought presents the plant with quite a different problem and production of compatible solutes, such as proline, is one strategy to ameliorate the effects of a low water potential. As mitochondria are involved in proline turnover it is likely that homeostatic balance of this osmolyte requires the coordinated action of multiple organelles (Atkin and Macherel, 2009).

Mitochondria in maturing and germinating seeds experience quite unique conditions. The water content during the latematuration phase is very low and a late-embryogenesis-abundant (LEA) protein is induced to protect mitochondrial membranes (Macherel et al., 2007; Tolleter et al., 2007). At the same time, the mitochondria presumably have to cope with hypoxic conditions caused by their own activity (Borisjuk and Rolletschek, 2009).

\section{PROTEOMICS OF MITOCHONDRIA FROM NON-GREEN TISSUES}

The most comprehensive proteomic studies in nonphotosynthetic tissues have been performed on mitochondria from Arabidopsis suspension cells, a de-differentiated cell type that, when grown in darkness with sugar, is non-photosynthetic. For this reason and space considerations, this discussion of proteomics and post-translational modifications will focus mainly on Arabidopsis suspension cells and etiolated rice seedlings.

Bioinformatic analysis of the Arabidopsis genome, using primarily prediction algorithms, has estimated that mitochondria may contain as many as 2000-3000 different proteins (Millar et al., 2005, 2006; Cui et al., 2011). It is surprising then that nearly 20 years into the proteomics era no more than 500-600 different mitochondrial isolation proteins have been experimentally confirmed from any plant mitochondrial source! This is not due to limitations in technology, as it is now routine to not only identify, but quantify, at least 1900 proteins from a single biological sample using standard tandem mass spectrometry (e.g., Balbuena et al., 2012). We therefore expect the gap between the number of experimental and predicted mitochondrial proteins to shrink in the coming years.

The most comprehensive proteomic characterization of purified mitochondria from non-photosynthetic cells has been from 
Arabidopsis suspension cells (Heazlewood et al., 2004). A total of 390 unique proteins were qualitatively identified by shotgun, reversed-phase (RP) LC-MS/MS analysis of 15-20 $\mu \mathrm{g}$ of tryptic peptides. Diverse groups of proteins were identified including proteins involved in energy and metabolism, DNA replication, transcription, translation, protein complex assembly, and signaling, as well as approximately 70 proteins of unknown function. Interestingly, various glycolytic enzymes were detected in mitochondrial preparations from Arabidopsis suspension cells (Heazlewood et al., 2004). Subsequent studies verified that these enzymes are indeed associated with mitochondria through scaffold proteins, such as the voltage-dependent anion channel (VDAC) located in the outer membrane (Graham et al., 2007). Additionally, in vivo association of cytosolic glycolytic enzymes with mitochondria is a dynamic process allowing for respiration to be supported in a dedicated manner consistent with substrate channeling (Graham et al., 2007). Although many of the individual subunits of the five respiratory complexes were identified by Heazlewood et al. (2004), follow-up proteomic analysis of these complexes was necessary to reveal the full protein complements (Meyer et al., 2008; Klodmann and Braun, 2011). In a study focusing on mitochondrial protein complexes, (Klodmann et al., 2011) identified 471 non-redundant proteins belonging to at least 35 different protein complexes. Subsequent proteomic analysis of purified outer membranes also expanded the compendium of proteins mapped to these mitochondrial subcompartment especially integral membrane proteins (Duncan et al., 2011; Tan et al., 2012).

Besides Arabidopsis roots and suspension cells, mitochondria from etiolated rice shoots have also been characterized at the proteome level (Bardel et al., 2002; Huang et al., 2009). Using both 2D gel electrophoresis and RP-LC-MS/MS analyses a total of 322 non-redundant proteins were identified in a non-quantitative manner. Comparison with Arabidopsis cell culture mitochondria (Heazlewood et al., 2004) revealed a similar cohort of proteins, although $20 \%$ of the rice mitochondrial proteins did not produce orthologous matches to the Arabidopsis mitochondrial proteome (Huang et al., 2009). And like the Arabidopsis experimental proteome, approximately $60 \%$ of rice mitochondrial proteins were predicted to be targeted to this organelle using various organelle prediction algorithms. Despite the wealth of shotgun and targeted mitochondrial proteomic studies it is obvious that a comprehensive compendium of experimentally-verified plant mitochondrial proteins is currently unavailable. This is apparent when one not only considers the gap between predicted and experimentally identified proteins, but also from perusing the current catalogue of approximately 500 mapped proteins. Missing from this list are many essential plant mitochondrial activities including regulatory proteins, transcription factors, metabolite translocators, and the wealth of tRNA synthases and pentratricopeptide repeat proteins. For example, the two regulatory enzymes of the pyruvate dehydrogenase complex (PDC) — the PDC kinase and phosphoPDC phosphatase, which are activities in mitochondria from both green and non-green tissues (Thelen et al., 1998a,b) - are also undetected in global plant mitochondrial proteomic studies.

While the early stages of non-photosynthetic mitochondrial proteome characterization dealt with protein cataloguing, more recent research has shifted toward comparative analyses to discover dynamic changes in mitochondrial protein expression. Proteomic comparisons of mitochondria from non-photosynthetic Arabidopsis suspension cells and developing photosynthetic shoots (Lee et al., 2008) as well as developing Arabidopsis roots and photosynthetic shoots (Lee et al., 2011) collectively revealed differences in TCA cycle and photorespiratory enzymes. In both instances, most of the component enzymes of the glycine decarboxylase complex, except for the lipoamide dehydrogenase (E3) subunit, which is shared with the PDC, were highly upregulated in green shoot mitochondria compared to the two non-photosynthetic counterparts. Additionally, FDH was highly induced in mitochondria from green shoots compared to roots and suspension cells. In contrast, three subunits of the PDC complex including E3 were more prominently expressed in root and suspension cell mitochondria. These and other changes to TCA and amino acid metabolism between mitochondria from green and non-green sources confirmed previous observations (e.g., upregulation of GDC in photorespiring tissues) but also suggested previously unknown differences in both carbon import/export and oxidative metabolism for this organelle as a direct result of photosynthetic capacity.

Additional comparative proteomic studies have been performed with mitochondria from etiolated rice shoots, analyzing the effect of a hypoxic/anoxic environment (Millar et al., 2004; Howell et al., 2007). In the absence of oxygen, mitochondrial respiration was impaired due to lower activity and expression of respiratory complexes cytochrome $\mathrm{bc}_{1}$ and cytochrome coxidase (Millar et al., 2004). Additionally, the E1 $\beta$ subunit of the PDC and a putative succinyl-CoA ligase (GDP-forming) $\beta$-chain as well as mitochondrial processing peptidase $\alpha$ - and $\beta$-chain subunits (a component of the cytochrome $b_{1}$ complex) were reduced in expression (Howell et al., 2007). In contrast, a TIM subunit was highly induced under anaerobic conditions. It was concluded that under anoxic conditions a direct link between respiratory capacity and protein import could be established at the cytochrome $\mathrm{bc}_{1}$ complex of the ETC. While total proteome coverage was not attained in either of these studies, the results illustrate the potential for comparative proteomics to elucidate the dynamic properties of mitochondria.

\section{PROTEIN PHOSPHORYLATION IN PLANT MITOCHONDRIA}

Several hundred posttranslational modifications (PTMs) - the covalent addition of a chemical group to amino acids in proteinsare known. They often lead to alterations in properties and function of the proteins and are therefore involved in the regulation of large variety of important biological processes (Wold, 1981; Mann and Jensen, 2003). The most well-studied PTM is phosphorylation, which is catalyzed by protein kinases (PKAs) while the dephosphorylation is catalyzed by protein phosphatases. This modification primarily targets the hydroxyl groups of Ser, Thr, and Tyr residues and has long been known to be a key player in signaling. Both protein phosphorylation and other PTMs such as acetylation are important in mammalian mitochondria (Guan and Xiong, 2011; Koc and Koc, 2012), but in plant mitochondria only protein phosphorylation has been studied in any detail. 
Table 1 | Phosphoproteins and phosphosites in plant mitochondria.

\begin{tabular}{|c|c|c|c|c|}
\hline No & Protein & Plant species & Phosphorylation site & References \\
\hline \multicolumn{5}{|c|}{ ENERGY } \\
\hline 1 & Pyruvate dehydrogenase (EC 1.2.4.1), E1 $\alpha$-subunit & $\begin{array}{l}\text { Potato } \\
\text { Arabidopsis } \\
\text { Pea } \\
\text { Rice }\end{array}$ & $\begin{array}{l}\text { Ser }^{298}: \text { YHGH(S)MSDP } \\
\text { Ser }^{292}: \text { YHGH(S)MSDP } \\
\text { Ser }{ }^{110}: \text { AITR(S)D(S)॥ } \\
\text { Ser }^{112}: \operatorname{TR}(S) D(\mathbf{S}) \| T A \\
\operatorname{Ser}^{293}: \text { YHGH(S)M(S)D } \\
\text { Ser }^{295}: \text { GH(S)M(S)DPGS }\end{array}$ & $\begin{array}{l}1,3,4^{a} \\
5^{a}, 10^{a}, 9^{a}, 11^{a} \\
14 \\
12^{a}\end{array}$ \\
\hline 2 & Aconitate hydratase (EC 4.2.1.3) & Potato & & 3 \\
\hline 3 & NAD-isocitrate dehydrogenase (EC 1.1.1.41) & Potato & & 3 \\
\hline 4 & Succinyl-CoA-ligase (EC 6.2.1.5), $\alpha$-subunit & $\begin{array}{l}\text { Potato } \\
\text { Arabidopsis } \\
\text { Rice }\end{array}$ & $\begin{array}{l}\text { Ser }^{304}: \text { GAIV(S)GGKG } \\
\text { Ser }{ }^{289}: \text { GAIV(S)GGKG } \\
\text { Ser }^{312}: \text { TVVE(S)PAKI }\end{array}$ & $\begin{array}{l}3 \\
9^{a}, 12^{a} \\
12^{a}\end{array}$ \\
\hline 5 & Succinyl-CoA-ligase (EC 6.2.1.5), $\beta$-subunit & Potato & & 3 \\
\hline 6 & Succinate dehydrogenase (EC 1.3.5.1), flavoprotein & $\begin{array}{l}\text { Potato } \\
\text { Arabidopsis } \\
\text { Rice }\end{array}$ & $\operatorname{Ser}^{161}:$ FGGQ(S)LDFG & $\begin{array}{l}3 \\
5 \\
12^{\mathrm{a}}\end{array}$ \\
\hline 7 & Succinate dehydrogenase (EC 1.3.5.1), subunit 5 & Arabidopsis & & 5 \\
\hline 8 & Malate dehydrogenase (EC 1.1.1.37) & $\begin{array}{l}\text { Potato } \\
\text { Arabidopsis } \\
\text { Rice }\end{array}$ & $\begin{array}{l}\operatorname{Ser}^{23}: \operatorname{RRSF}(\mathbf{S})(S) G S V \\
\operatorname{Ser}^{24}: \operatorname{RSF}(S)(\mathbf{S}) G S V P \\
\operatorname{Ser}^{314}: \text { LGPL(S)DFEK } \\
\text { Ser }^{313}: \text { LGQL(S)DFEK }\end{array}$ & $\begin{array}{l}3 \\
9^{\mathrm{a}} \\
12^{\mathrm{a}}\end{array}$ \\
\hline 12 & $\begin{array}{l}\text { Complex III (EC 1.10.2.2), } \beta \text {-mitochondria processing } \\
\text { peptidase subunit }\end{array}$ & $\begin{array}{l}\text { Potato } \\
\text { Rice }\end{array}$ & $\begin{array}{l}\text { Ser }^{485}: \operatorname{SWFR}(\mathbf{S}) \text { HTY(S) } \\
\text { Ser }^{489}: \text { (S)HTY(S)DDEF }\end{array}$ & $\begin{array}{l}3 \\
12^{\mathrm{a}}\end{array}$ \\
\hline 13 & $\begin{array}{l}\text { Protein mitochondrial-processing peptidase } \\
\text { (EC 3.4.24.64), } \alpha \text {-subunit }\end{array}$ & Rice & $\begin{array}{l}\operatorname{Ser}^{234}: \operatorname{HRLD}(\mathbf{S})(\mathrm{S}) I L E \\
\operatorname{Ser}^{235}: \operatorname{RLD}(\mathrm{S})(\mathbf{S}) \text { ILEE }\end{array}$ & $12^{\mathrm{a}}$ \\
\hline 14 & $\mathrm{~F}_{1}$ ATPase (EC 3.6.1.3), $\delta$-subunit & Potato & & 2 \\
\hline 15 & ATP synthase (EC 3.6.1.3), $\alpha$-subunit & Potato & & 3 \\
\hline 16 & ATP synthase (EC 3.6.1.3), $\beta$-subunit & Rice & $\begin{array}{l}\text { Ser }^{355}: \text { GRIP(S)AVGY } \\
\text { Thr }{ }^{362}: \text { GYQP(T)LATD } \\
\text { Ser }\end{array}$ & $12^{\mathrm{a}}$ \\
\hline 17 & $F_{0}$ - ATPase (EC 3.6.1.3), b/C/D-subunit & $\begin{array}{l}\text { Potato } \\
\text { Rice } \\
\text { Arabidopsis } \\
\text { Arabidopsis }\end{array}$ & $\begin{array}{l}\operatorname{Ser}^{157}: \operatorname{KAVD}(\mathbf{S}) \text { LVPI } \\
\operatorname{Ser}^{77}: \operatorname{VENK}(\mathbf{S}) \text { QGSE } \\
\text { Ser }^{80}: \operatorname{K}(\mathrm{S}) \text { QG(S)EVLL } \\
\text { Ser }^{386}: \text { EKSE(S)LEEI } \\
\text { Ser }^{248}: \text { QRGV(T)LGDP }\end{array}$ & $\begin{array}{l}2 \\
12^{\mathrm{a}} \\
9^{\mathrm{a}}\end{array}$ \\
\hline
\end{tabular}


Table 1 | Continued

\begin{tabular}{|c|c|c|c|c|}
\hline No & Protein & Plant species & Phosphorylation site & References \\
\hline \multirow[t]{3}{*}{18} & \multirow[t]{3}{*}{ Formate DH (EC 1.2.1.2) } & \multirow[t]{2}{*}{ Potato } & Thr $76:$ QYIV(T)PDKE & \multirow[t]{2}{*}{$3,4^{a}$} \\
\hline & & & Thr ${ }^{333}:$ NQAM(T)PHIS & \\
\hline & & \multicolumn{2}{|l|}{ Arabidopsis } & 5 \\
\hline 19 & Glutamate DH 1 (EC 1.4.1.2) & \multicolumn{2}{|l|}{ Arabidopsis } & 5 \\
\hline 20 & Glutamate DH 2 (EC 1.4.1.2) & \multicolumn{2}{|l|}{ Arabidopsis } & 5 \\
\hline 21 & Mitochondrial lipoamide dehydrogenase 1 (EC 1.8.1.4) & Arabidopsis & Ser $319:$ TPFT(S)GLDL & $9^{a}, 12^{a}$ \\
\hline \multicolumn{5}{|c|}{ METABOLISM } \\
\hline 22 & Protein cysteine synthase (EC 2.5.1.47) & Rice & $\operatorname{Ser}^{130}:$ PAGY(S)LDKO & $12^{\mathrm{a}}$ \\
\hline \multicolumn{5}{|c|}{ PROTEIN FATE } \\
\hline \multirow[t]{2}{*}{23} & \multirow[t]{2}{*}{ Heat shock protein 90} & \multirow{2}{*}{$\begin{array}{l}\text { Potato } \\
\text { Arabidopsis }\end{array}$} & & \multirow{2}{*}{$\begin{array}{l}3 \\
5^{a}, 9^{a}, 10^{a}, 11^{a}\end{array}$} \\
\hline & & & Ser $^{219}:$ EKEI(S)DDEE & \\
\hline \multirow[t]{9}{*}{24} & \multirow[t]{5}{*}{ Heat shock protein 70} & \multirow{5}{*}{$\begin{array}{l}\text { Potato } \\
\text { Bean } \\
\text { Rice }\end{array}$} & & \multirow{5}{*}{$\begin{array}{l}3 \\
6 \\
12^{a}\end{array}$} \\
\hline & & & & \\
\hline & & & $\operatorname{Ser}^{419}:$ GLSE(S)DIEK & \\
\hline & & & Ser ${ }^{526}:$ GSSS(S)(S)GGD & \\
\hline & & & $\mathrm{Ser}^{527}: \mathrm{SSS}(\mathrm{S})(\mathbf{S}) \mathrm{GGDO}$ & \\
\hline & \multirow[t]{3}{*}{ Heat shock protein 70-1 } & \multirow[t]{3}{*}{ Arabidopsis } & Ser ${ }^{398}$ : OEIV(S)EIFGK & \multirow[t]{3}{*}{$9^{a}$} \\
\hline & & & Ser $^{404}:$ IFGK(S)PCKG & \\
\hline & & & Ser ${ }^{552}:$ SGGL(S)DDEI & \\
\hline & Heat shock protein $70-2$ & Arabidopsis & Ser ${ }^{409}:$ IFGK(S)PSKG & $12^{a}$ \\
\hline \multirow[t]{2}{*}{25} & Chaperonin 60 & \multicolumn{2}{|l|}{ Potato } & 3 \\
\hline & 60 kDa chaperonin 7 & Rice & $\begin{array}{l}\text { Ser }^{137}: \text { OSYG(S)PKV } \\
\text { Thr }^{522}: \text { DKLP(T)ANFD }\end{array}$ & $12^{a}$ \\
\hline
\end{tabular}

\begin{tabular}{|c|c|c|c|c|}
\hline \multirow[t]{2}{*}{26} & Mitochondrial small heat shock protein & Potato & & 3 \\
\hline & Mitochondrial small heat shock protein, 22 kDa & $\begin{array}{l}\text { Maize } \\
\text { Rice }\end{array}$ & $\begin{array}{l}\operatorname{Ser}^{58}: \text { GRLL(S)LMDD } \\
\operatorname{Ser}^{101}: \operatorname{ERDE}(\mathbf{S}) \text { DDDS }\end{array}$ & $\begin{array}{l}7 \\
12^{a}\end{array}$ \\
\hline 27 & $26.2 \mathrm{kDa}$ heat shock protein & Rice & $\begin{array}{l}\text { Tyr }^{71}: \operatorname{PLRR}(Y) D W V D \\
\text { Ser }^{77}: \operatorname{VDE}(\mathbf{S}) \mathrm{G}(\mathrm{T}) \mathrm{D}(\mathrm{S}) \\
\text { Thr }^{79}: \mathrm{DE}(\mathrm{S}) \mathrm{G}(\mathrm{T}) \mathrm{D}(\mathrm{S}) \mathrm{GD} \\
\mathrm{Ser}^{81}: \text { (S)G(T)D(S)GDE(Y) } \\
\text { Tyr }^{85}: \text { (S)GDE(Y)DA(T)D } \\
\text { Thr }^{88}: \text { E(Y)DA(T)DDGR }\end{array}$ & $12^{\mathrm{a}}$ \\
\hline 28 & GRPE co-chaperone & Arabidopsis & & 5 \\
\hline 29 & Protein GrpE 1 & Rice & Ser $^{81}:$ SPEL(S)DKEE & $12^{\mathrm{a}}$ \\
\hline 30 & Lon1 protease (EC 3.4.21.53) & Arabidopsis & $\mathrm{Ser}^{74}$ : KAVE(S)DSEV & $5^{a}, 9^{a}$ \\
\hline \multicolumn{5}{|c|}{ TRANSCRIPTION } \\
\hline 31 & $\begin{array}{l}\text { Mitochondrial transcription termination factor family } \\
\text { protein [At1g62010] }\end{array}$ & Arabidopsis & $\mathrm{Ser}^{53}:$ FKSS(S)FLDS & $12^{\mathrm{a}}$ \\
\hline 32 & $\begin{array}{l}\text { Transcription termination factor domain-containing } \\
\text { protein [AT2G44020] }\end{array}$ & Arabidopsis & $\begin{array}{l}\text { Thr }{ }^{488}: \text { GEIV(T)DEEE } \\
\text { Ser }^{495}: \text { EEDE(S)DDEV }\end{array}$ & $12^{\mathrm{a}}$ \\
\hline
\end{tabular}


Table 1 | Continued

\begin{tabular}{|c|c|c|c|c|}
\hline No & Protein & Plant species & Phosphorylation site & References \\
\hline 33 & $\begin{array}{l}\text { Mitochondrial transcription termination factor family } \\
\text { protein [At2g21710] }\end{array}$ & Arabidopsis & $\begin{array}{l}\text { Tyr }{ }^{501}: \text { DEVL(Y)RRTL } \\
\text { Tyr }{ }^{168}: \text { FGIT(Y)A(T)NV } \\
\text { Thr }{ }^{170:} \text { IT(Y)A(T)NVTD }\end{array}$ & $9^{a}$ \\
\hline
\end{tabular}

\section{RNA PROCESSING}

\begin{tabular}{|c|c|c|c|c|}
\hline 34 & $\begin{array}{l}\text { Putative mitochondrial RNA helicase (EC 3.6.4.13), } \\
\text { helicase } 2\end{array}$ & Arabidopsis & Ser603: GGRS(S)FGGF & $12^{\mathrm{a}}$ \\
\hline 35 & $\begin{array}{l}\text { Protein ATP-dependent RNA helicase (EC 3.6.4.13), } \\
\text { SUV3 }\end{array}$ & Rice & Ser693: VEQA(S)DDNA & $12^{\mathrm{a}}$ \\
\hline
\end{tabular}

\section{PROTEIN SYNTHESIS}

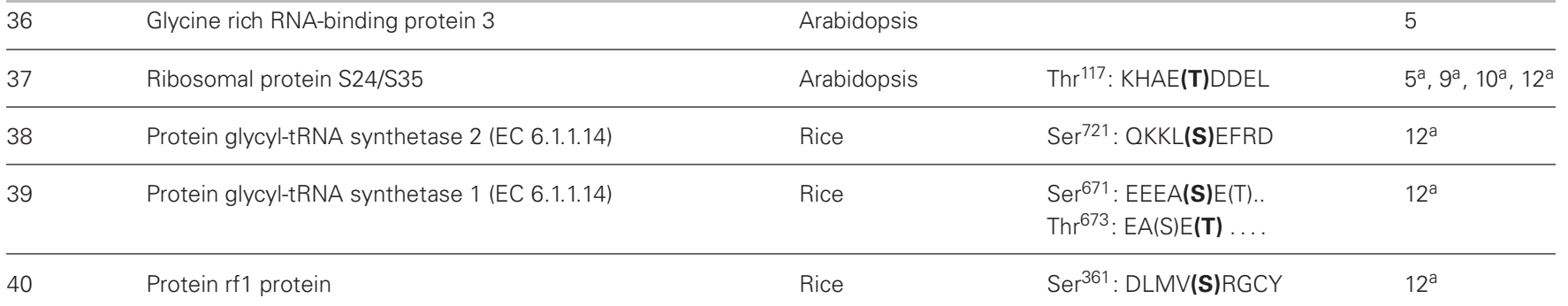

\section{DEFENSE, STRESS, DETOXIFICATION}

$41 \quad$ Mn superoxide dismutase (1.15.1.1)

Potato

3

\section{COMMUNICATION/SIGNALING}

\begin{tabular}{|c|c|c|c|c|}
\hline 42 & MAM protein & Arabidopsis & Ser $^{94}:$ DNID(S)DEEM & 5 \\
\hline 43 & MAM 33 & Arabidopsis & & 5 \\
\hline 44 & $\begin{array}{l}\text { Protein calcium-binding mitochondrial protein } \\
\text { Anon-60Da }\end{array}$ & Rice & Ser $^{609}:$ ASAS(S)VSKE & $12^{a}$ \\
\hline 45 & Protein protein-tyrosine phosphatase 1 (EC 3.1.3.48) & Rice & $\operatorname{Ser}^{269}:$ CLIP(S)LK.. & $12^{a}$ \\
\hline \multicolumn{5}{|c|}{ TRANSPORT } \\
\hline 46 & $\begin{array}{l}\text { Mitochondrial glycoprotein family protein } \\
\text { [AT4G32605.1] }\end{array}$ & Arabidopsis & $\begin{array}{l}\text { Ser }^{76}: \text { SLEG(S)(T)GAV } \\
\text { Thr }^{77}: \text { LEG(S)(T)GAVL }\end{array}$ & $9^{a}$ \\
\hline 47 & $\begin{array}{l}\text { Mitochondrial glycoprotein family protein } \\
\text { [AT2G39795] }\end{array}$ & Arabidopsis & Ser $^{84}:$ DNID(S)DEEM & $5^{\mathrm{a}}, 12^{\mathrm{a}}$ \\
\hline 48 & $\begin{array}{l}\text { Protein mitochondrial glycoprotein, expressed } \\
\text { [BAD33248.1] }\end{array}$ & Rice & $\begin{array}{l}\text { Ser } \\
\text { 88: } \\
\text { Ser }^{96}: \text { CIVQ(S)EE(S)EIEC }\end{array}$ & $12^{\mathrm{a}}$ \\
\hline 49 & $\begin{array}{l}\text { Protein mitochondrial glycoprotein, expressed } \\
\text { [A2ZP51.1] }\end{array}$ & Rice & Ser $^{81}:$ FAEE(S)DDHD & $12^{a}$ \\
\hline 50 & $\begin{array}{l}\text { Protein mitochondrial glycoprotein, expressed } \\
\text { [BAF25817.1] }\end{array}$ & Rice & Ser ${ }^{115}: \operatorname{RRVE(S)LERG~}$ & $12^{\mathrm{a}}$ \\
\hline 51 & Mitochondrial substrate carrier family protein & Arabidopsis & Ser $^{64}:$ RGNN(S)FSTO & $12^{\mathrm{a}}$ \\
\hline 52 & $\begin{array}{l}\text { Mitochondrial import inner membrane translocase, } \\
\text { subunit TIM17/TIM22/TIM23 }\end{array}$ & Arabidopsis & Ser $^{15}:$ DPSS(S)PPPI & $12^{a}$ \\
\hline \multirow[t]{2}{*}{53} & $\begin{array}{l}\text { Mitochondrial import inner membrane translocase, } \\
\text { subunit TIM23 }\end{array}$ & Rice & $\begin{array}{l}\text {Ser}^{8}: \operatorname{PRLF}(\mathbf{S})(S) G(S) G \\
\text { Ser }^{9}: \operatorname{RLF}(S)(\mathbf{S}) G(S) G S \\
\text { Ser }^{11}: F(S)(S) G(\mathbf{S}) G T R D\end{array}$ & $12^{a}$ \\
\hline & & Arabidopsis & $\begin{array}{l}\text { Ser }{ }^{126}: \text { AGIE(S)GVVA } \\
\text { Ser }^{140:} \text { DVWT(S)VVAG }\end{array}$ & $13^{a}$ \\
\hline
\end{tabular}




\section{Table 1 | Continued}

\begin{tabular}{|c|c|c|c|c|}
\hline No & Protein & Plant species & Phosphorylation site & References \\
\hline \multirow{9}{*}{55} & & & $\mathrm{Ser}^{73}:$ OYGR(S)PASL & $9^{a}, 12^{a}$ \\
\hline & & & $\operatorname{Ser}^{76}: \mathrm{R}(\mathrm{S}) \mathrm{PA}(\mathbf{S}) \mathrm{LPDA}$ & $12^{\mathrm{a}}$ \\
\hline & & & $\operatorname{Ser}^{140}:$ VRPI(S)PKSP & $9^{\mathrm{a}}, 10^{\mathrm{a}}, 12^{\mathrm{a}}$ \\
\hline & & & $\mathrm{Thr}^{280}: \mathrm{SDP}(\mathrm{S})(\mathrm{T}) \mathrm{PKPN}$ & $12^{\mathrm{a}}$ \\
\hline & & Rice & Ser $^{94}:$ KPII(S)PAST & $12^{\mathrm{a}}$ \\
\hline & & & $\operatorname{Ser}^{126}:$ TIED(S)DDDD & \\
\hline & & & $\operatorname{Ser}^{178}:$ IRSO(S)ATG(S) & \\
\hline & & & $\operatorname{Ser}^{182}:$ (S)ATG(S)LHGA & \\
\hline & & & Thr 256: TDPS(T)PKPN & \\
\hline 58 & $\begin{array}{l}\text { Protein mitochondrial 2-oxoglutarate/malate carrier } \\
\text { protein }\end{array}$ & Rice & $\operatorname{Ser}^{97}:$ ARLG(S)FRVL & $12^{\mathrm{a}}$ \\
\hline 59 & Protein mitochondrial carrier protein & Rice & Ser ${ }^{419}$ : ARAN(S)IEVS & $12^{\mathrm{a}}$ \\
\hline 60 & Mitochondrial carrier protein & Arabidopsis & Ser $^{155}$ : AAPE(S)PSSN & $5^{a}$ \\
\hline 61 & TraB family protein & Arabidopsis & $\operatorname{Ser}^{64}:$ DSVF(S)GGDG & $12^{\mathrm{a}}$ \\
\hline
\end{tabular}

\section{STRUCTURE ORGANIZATION}

62 Protein inner membrane protein OXA1L

Rice

$\operatorname{Ser}^{422}$ : SQRF(S)DLEN

$12^{\mathrm{a}}$

\section{MISCELLANEOUS}

\begin{tabular}{lllll}
63 & Stomatin-like protein & Arabidopsis & Thr $^{362:}$ ALDE(T)DLEE & Rice \\
\hline 64 & Prohibitin & Rice
\end{tabular}

This list has been compiled by first manually going through the phosphoproteomic literature on non-photosynthetic plant organisms published before 2008. Secondly, the identified proteins were used as search input in the Plant Protein Phosphorylation Database and Yao et al. (2012) - http://p3db.org/- resulting new phosphosites were included in the list. Lastly, the mitochondrial proteins from Duncan et al. (2011) were searched against P DB and the hits included. The blank spaces in the column labeled Phosphorylation site indicate that the phosphoprotein, but not the specific site of phosphorylation was identified.

All phosphorylation sites are highlighted with parentheses. The specific phosphorylation site is highlighted with bold.

a This reference includes phosphosite information.

1. (Sommarin et al., 1990); 2, (Struglics et al., 1998); 3, (Bykova et al., 2003a); 4, (Bykova et al., 2003b); 5, (Ito et al., 2009); 6, (Vidal et al., 1993); 7, (Lund et al., 2001); 8, (Takahashi et al., 2003); 9, (Sugiyama et al., 2008); 10, (Li et al., 2009); 11, (de la Fuente van Bentem et al., 2008); 12, (Nakagami et al., 2010); 13, (Meyer et al., 2012); 14, (Budde and Randall, 1990).

Traditionally, the methods for detecting phosphoproteins are based on incorporation of radioactive phosphate $\left({ }^{32} \mathrm{P}\right)$. This is either done by in vitro phosphorylation reactions using protein extracts or isolated organelles or by a more physiologically relevant in vivo approach using cells. The phosphorylated proteins are then typically separated by gel electrophoresis and detected by phosphoimaging or autoradiography (Thelen et al., 2000; Bykova et al., 2003a; Berwick and Tavare, 2004). A fluorescent phosphosensor dye called Pro-Q Diamond has also been developed (Schulenberg et al., 2003; Ito et al., 2009). The phosphoproteins can be cut out of the gel and identified by LC-MS/MS (Pappin et al., 1993; Heazlewood et al., 2004; Ito et al., 2009). However, more recently shotgun methods of identifying phosphopeptides and phosphoproteins first uses a phosphopeptide enrichment method such as immobilized metal affinity chromatography (IMAC) (Posewitz and Tempst, 1999; Stensballe et al., 2001) or titanium oxide chromatography (MOC) followed by LC-MS/MS. In this way thousands of phosphorylation sites can be identified without the use of gel electrophoresis (e.g., Engholm-Keller et al., 2012; Meyer et al., 2012).

To date, 64 plant mitochondrial proteins have been reported to be phosphorylated on 103 phosphosites (Table 1). The distribution of these on Ser, Thr, and Tyr residues is 81, 14, and 5\%, respectively, or close to the distribution in all the known 22,995 eukaryotic phosphoproteins with 100,281 phosphosites (Rao and Møller, 2012) in spite of the fact that plants do not contain canonical Tyr kinases (Yao et al., 2012). 
The largest group of phosphorylated proteins is energy and transport (Table 1), a trend also observed in yeast (Reinders et al., 2007). Phosphorylation sites have been identified on most of the TCA cycle enzymes, and on complexes III-V, whereas no phosphoproteins have been found in Complexes I and II. It seems likely that oxidative phosphorylation is regulated by reversible protein phosphorylation. Other major processes that may be regulated by phosphorylation are transcription, translation, protein folding, and metabolite transport across the inner mitochondrial membrane. In mammalian mitochondria, translation is regulated by protein phosphorylation (Koc and Koc, 2012).

PDC, which produces the acetyl-CoA entering the TCA cycle, is the only enzyme in plant mitochondria where the detailed regulation by reversible phosphorylation/dephosphorylation has been elucidated (Rubin and Randall, 1977; Budde and Randall, 1990). When PDC products accumulate, they activate the PDC kinase, which phosphorylates and inactivates the PDH component of PDC (Tovar-Mendez et al., 2003). In contrast, PDC substrates inhibit the PDC kinase and stimulate the phosphoPDC phosphatase to dephosphorylate $\mathrm{PDH}$ and give a higher activity (Randall et al., 1981; Schuller and Randall, 1990; Moore et al., 1993). FDH phosphorylation appears to be regulated in a similar way by some of the same metabolites, but the effect on FDH activity is still unknown (Bykova et al., 2003b).

In yeast, phosphorylation of TOM22, Mim1, and TOM79 by casein kinase 2 (CK2) and PKA have been shown to regulate the function of TOM. CK2 promotes biogenesis of specific TOM complexes and CK2 inhibits specific TOM receptor activities and thereby the import of mitochondrial metabolite carriers. Together these phosphorylations regulate protein homeostasis (Schmidt et al., 2011). Another study has shown that phosphorylation of subunit $g$ of the yeast ATP synthase subunit $\mathrm{F}_{o}$ inhibits

\section{REFERENCES}

Ambard-Bretteville, F., Sorin, C., Rebeille, F., Hourton-Cabassa, C., and Colas Des Francs-Small, C. (2003). Repression of formate dehydrogenase in Solanum tuberosum increases steady-state levels of formate and accelerates the accumulation of proline in response to osmotic stress. Plant Mol. Biol. 52, 1153-1168.

Atkin, O. K., and Macherel, D. (2009). The crucial role of plant mitochondria in orchestrating drought tolerance. Ann. Bot. 103, 581-597.

Balbuena, T. S., He, R., Salvato, F., Gang, D. R., and Thelen, J. J. (2012). Large-scale proteome comparative analysis of developing rhizomes of the ancient vascular plant equisetum hyemale. Front. Plant Sci. 3:131. doi: 10.3389/fpls.2012.00131

Bardel, J., Louwagie, M., Jaquinod, M., Jourdain, A., Luche, S., Rabilloud, T., et al. (2002). A survey of the plant mitochondrial proteome in relation to development. Proteomics 2, 880-898.
Berwick, D. C., and Tavare, J. M. (2004). Identifying protein kinase substrates: hunting for the organgrinder's monkeys. Trends Biochem. Sci. 29, 227-232.

Borisjuk, L., and Rolletschek, $\mathrm{H}$. (2009). The oxygen status of the developing seed. New Phytol. 182, 17-30.

Budde, R. J., and Randall, D. D. (1990). Pea leaf mitochondrial pyruvate dehydrogenase complex is inactivated in vivo in a light-dependent manner. Proc. Natl. Acad. Sci. U.S.A. 87, 673-676.

Bykova, N. V., Egsgaard, H., and Møller, I. M. (2003a). Identification of 14 new phosphoproteins involved in important plant mitochondrial processes. FEBS Lett. 540, 141-146.

Bykova, N. V., Stensballe, A., Egsgaard, H., Jensen, O. N., and Møller, I. M. (2003b). Phosphorylation of formate dehydrogenase in potato tuber mitochondria. J. Biol. Chem. 278, 26021-26030.

Colas Des Francs-Small, C., AmbardBretteville, F., Small, I. D., and

dimerization of the ATP synthase and is thus involved in the regulation of the bioenergetic state of mitochondria (Reinders et al., 2007). Phosphorylated TOM and ATP synthase $\mathrm{F}_{o}$ subunits have also been found in plant mitochondria from non-photosynthetic cells (Table 1) and the function of these phosphorylations might be similar to that in yeast. Finally, the interaction of chaperone HSP90 with co-chaperones is regulated by phosphorylation in mammalian mitochondria (Mollapour et al., 2011; Johnson, 2012). HSP90 is one of the identified phosphoproteins in plant mitochondria (Table 1), but the site of phosphorylation so far identified is different from that of mammalian HSP90 (Mollapour et al., 2011), so the regulatory mechanism may not be the same.

Considering that plant mitochondria contain so many phosphoproteins, we might expect to find a substantial number of PKAs in plant mitochondria as well as a somewhat smaller number of protein phosphatases (Juszczuk et al., 2007). However, even in the most extensive proteomic studies published to date only ten protein kinases have been identified and no protein phosphatases (Heazlewood et al., 2004; Duncan et al., 2011; Taylor et al., 2011). This may indicate that each enzyme is present in very few copies so that they are below the technical detection limit. Alternatively there are actually relatively few kinases and phosphatases each with a relatively broad specificity. Future in depth proteomic studies will no doubt give us more information about such regulatory pathways.

\section{ACKNOWLEDGMENTS}

This study was supported by grants from the Danish Council for Independent Research—Natural Sciences (to Ian M. Møller) and a 2012 Sabbatical fellowship (to Jay J. Thelen) from the OECD Cooperative Research Programme: Biological Resource Management for Sustainable Agricultural Systems.

Remy, R. (1993). Identification of a major soluble-protein in mitochondria from nonphotosynthetic tissues as nad-dependent formate dehydrogenase. Plant Physiol. 102, 1171-1177.

Considine, M. J., Goodman, M., Echtay, K. S., Laloi, M., Whelan, J., Brand, M. D., et al. (2003). Superoxide stimulates a proton leak in potato mitochondria that is related to the activity of uncoupling protein. J. Biol. Chem. 278, 22298-22302.

Cui, J. A., Liu, J. H., Li, Y. H., and Shi, T. L. (2011). Integrative identification of arabidopsis mitochondrial proteome and its function exploitation through protein interaction network. PLoS ONE 6:e16022. doi: 10.1371/journal.pone.0016022

de la Fuente van Bentem, S., Anrather, D., Dohnal, I., Roitinger, E., Csaszar, E., Joore, J., et al. (2008). Site-specific phosphorylation profiling of Arabidopsis proteins by mass spectrometry and peptide chip analysis. J. Proteom. Res. 7, 2458-2470.
Douce, R., Bonner, W. D., and Christen, E. L. (1972). Preparation of intact plant mitochondria. Biochim. Biophys. Acta 275, 148-160.

Douce, R., and Neuburger, M. (1989). The uniqueness of plant-mitochondria. Annu. Rev. Plant Physiol. Plant Mol. Biol. 40, 371-414.

Duncan, O., Taylor, N. L., Carrie, C. Eubel, H., Kubiszewski-Jakubiak, S., Zhang, B. T., et al. (2011). Multiple lines of evidence localize signaling, morphology, and lipid biosynthesis machinery to the mitochondrial outer membrane of arabidopsis. Plant Physiol. 157, 1093-1113.

Engholm-Keller, K., Birck, P., Storling, J., Pociot, F., Mandrup-Poulsen, T. and Larsen, M. R. (2012). TiSH a robust and sensitive global phosphoproteomics strategy employing a combination of $\mathrm{TiO}(2)$, SIMAC, and HILIC. J. Proteomics 75, 5749-5761.

Eubel, H., Lee, C. P., Kuo, J., Meyer, E. H., Taylor, N. L., and Millar, A. 
H. (2007). Free-flow electrophoresis for purification of plant mitochondria by surface charge. Plant J. 52, 583-594.

Fredlund, K. M., Rasmusson, A. G., and Møller, I. M. (1991). Oxidation of external $\mathrm{Nad}(\mathrm{P}) \mathrm{H}$ by purified mitochondria from fresh and aged red beetroots (Beta-Vulgaris L). Plant Physiol. 97, 99-103.

Geigenberger, P. (2003). Response of plant metabolism to too little oxygen. Curr. Opin. Plant Biol. 6, 247-256.

Graham, J. W. A., Williams, T. C. R., Morgan, M., Fernie, A. R., Ratcliffe, R. G., and Sweetlove, L. J. (2007). Glycolytic enzymes associate dynamically with mitochondria in response to respiratory demand and support substrate channeling. Plant Cell 19, 3723-3738.

Greenway, H., Armstrong, W., and Colmer, T. D. (2006). Conditions leading to high $\mathrm{CO}_{2}(>5 \mathrm{kPa})$ in waterlogged-flooded soils and possible effects on root growth and metabolism. Ann. Bot. 98, 9-32.

Guan, K. L., and Xiong, Y. (2011). Regulation of intermediary metabolism by protein acetylation. Trends Biochem. Sci. 36, 108-116.

Heazlewood, J. L., Tonti-Filippini, J. S., Gout, A. M., Day, D. A., Whelan, J., and Millar, A. H. (2004). Experimental analysis of the Arabidopsis mitochondrial proteome highlights signaling and regulatory components, provides assessment of targeting prediction programs, and indicates plantspecific mitochondrial proteins. Plant Cell 16, 241-256.

Howell, K. A., Cheng, K., Murcha, M. W., Jenkin, L. E., Millar, A. H., and Whelan, J. (2007). Oxygen initiation of respiration and mitochondrial biogenesis in rice. J. Biol. Chem. 282, 15619-15631.

Huang, S., Taylor, N. L., Narsai, R., Eubel, H., Whelan, J., and Millar, A. H. (2009). Experimental analysis of the rice mitochondrial proteome, its biogenesis, and heterogeneity. Plant Physiol. 149, 719-734.

Igamberdiev, A. U. (1999). Foundations of metabolic organization: coherence as a basis of computational properties in metabolic networks. Biosystems 50, 1-16.

Ito, J., Taylor, N. L., Castleden, I., Weckwerth, W., Millar, A. H., and Heazlewood, J. L. (2009). A survey of the Arabidopsis thaliana mitochondrial phosphoproteome. Proteomics 9, 4229-4240.

Johnson, J. L. (2012). Evolution and function of diverse Hsp90 homologs and cochaperone proteins. Biochim. Biophys. Acta 1823, 607-613.

Juszczuk, I. M., Bykova, N. V., and Møller, I. M. (2007). Protein phosphorylation in plant mitochondria. Physiol. Plant. 129, 90-103.

Klodmann, J., and Braun, H. P. (2011) Proteomic approach to characterize mitochondrial complex I from plants. Phytochemistry 72, 1071-1080.

Klodmann, J., Senkler, M., Rode, C., and Braun, H. P. (2011). Defining the protein complex proteome of plant mitochondria. Plant Physiol. $157,587-598$.

Koc, E. C., and Koc, H. (2012). Regulation of mammalian mitochondrial translation by post-translational modifications. Biochim. Biophys. Acta 1819, 1055-1066.

Kubo, T., and Newton, K. J. (2008). Angiosperm mitochondrial genomes and mutations. Mitochondrion 8, 5-14.

Laloi, M. (1999). Plant mitochondrial carriers: an overview. Cell. Mol. Life Sci. 56, 918-944.

Lee, C. P., Eubel, H., O’Toole, N., and Millar, A. H. (2008). Heterogeneity of the mitochondrial proteome for photosynthetic and non-photosynthetic Arabidopsis metabolism. Mol. Cell. Proteom. 7, 1297-1316.

Lee, C. P., Eubel, H., O'Toole, N., and Millar, A. H. (2011). Combining proteomics of root and shoot mitochondria and transcript analysis to define constitutive and variable components in plant mitochondria. Phytochemistry 72 1092-1108.

Li, H., Wong, W. S., Zhu, L., Guo, H. W., Ecker, J., and Li, N. (2009). Phosphoproteomic analysis of ethylene-regulated protein phosphorylation in etiolated seedlings of Arabidopsis mutant ein2 using two-dimensional separations coupled with a hybrid quadrupole time-of-flight mass spectrometer. Proteomics 9, 1646-1661.

Liden, A. C., and Møller, I. M. (1988). Purification, characterization and storage of mitochondria from Jerusalem artichoke tubers. Physiol. Plant. 72, 265-270.

Lind, C., Hallden, C., and Møller, I. M. (1991). Protein-synthesis in mitochondria purified from roots, leaves and flowers of sugar-beet. Physiol. Plant. 83, 7-16.

Lister, R., Hulett, J. M., Lithgow, T., and Whelan, J. (2005). Protein import into mitochondria: origins and functions today (Review). Mol. Membr. Biol. 22, 87-100.
Lund, A. A., Rhoads, D. M., Lund, A. L., Cerny, R. L., and Elthon, T. E. (2001). In vivo modifications of the maize mitochondrial small heat stress protein, HSP22. J. Biol. Chem. 276, 29924-29929.

Macherel, D., Benamar, A., AvelangeMacherel, M. H., and Tolleter, D. (2007). Function and stress tolerance of seed mitochondria. Physiol. Plant. 129, 233-241.

Mann, M., and Jensen, O. N. (2003). Proteomic analysis of posttranslational modifications. Nat. Biotech. 21, 255-261.

Meyer, E. H., Taylor, N. L., and Millar, A. H. (2008). Resolving and identifying protein components of plant mitochondrial respiratory complexes using three dimensions of gel electrophoresis. J. Proteome Res. 7, 786-794.

Meyer, L. J., Gao, J., Xu, D., and Thelen, J. J. (2012). Phosphoproteomic analysis of seed maturation in Arabidopsis, rapeseed, and soybean. Plant Physiol. 159, 517-528.

Millar, A. H., Heazlewood, J. L., Kristensen, B. K., Braun, H. P., and Møller, I. M. (2005). The plant mitochondrial proteome. Trends Plant Sci. 10, 36-43.

Millar, A. H., Sweetlove, L. J., Giege, P., and Leaver, C. J. (2001). Analysis of the Arabidopsis mitochondrial proteome. Plant Physiol. 127, 1711-1727.

Millar, A. H., Trend, A. E., and Heazlewood, J. L. (2004). Changes in the mitochondrial proteome during the anoxia to air transition in rice focus around cytochromecontaining respiratory complexes. J. Biol. Chem. 279, 39471-39478.

Millar, A. H., Whelan, J., and Small, I. (2006). Recent surprises in protein targeting to mitochondria and plastids. Curr. Opin. Plant Biol. 9, 610-615.

Mollapour, M., Tsutsumi, S., Truman, A. W., Xu, W., Vaughan, C. K., Beebe, K., et al. (2011). Threonine 22 phosphorylation attenuates Hsp90 interaction with cochaperones and affects its chaperone activity. Mol. Cell 41, 672-681.

Moore, A. L., Gemel, J., and Randall, D. D. (1993). The regulation of Pyruvate dehydrogenase activity in pea leaf mitochondria (the effect of respiration and oxidative phosphorylation). Plant Physiol. 103, 1431-1435.

Moreau, F., and Romani, R. (1982). Preparation of avocado mitochondria using self-generated percoll density gradients and changes in buoyant density during ripening. Plant Physiol. 70, 1380-1384.
Nakagami, H., Sugiyama, N., Mochida, K., Daudi, A., Yoshida, Y., Toyoda, T., et al. (2010). Large-scale comparative phosphoproteomics identifies conserved phosphorylation sites in plants. Plant Physiol. 153, 1161-1174.

Neuburger, M., Journet, E. P., Bligny, R., Carde, J. P., and Douce, R. (1982). Purification of plantmitochondria by isopycnic centrifugation in density gradients of percoll. Arch. Biochem. Biophys. 217, 312-323.

Palmieri, F., Rieder, B., Ventrella, A., Blanco, E., Do, P. T., Nunes-Nesi, A., et al. (2009). Molecular identification and functional characterization of Arabidopsis thaliana mitochondrial and chloroplastic NAD $(+)$ carrier proteins. J. Biol. Chem. 284, 31249-31259.

Pappin, D. J., Hojrup, P., and Bleasby, A. J. (1993). Rapid identification of proteins by peptide-mass fingerprinting. Curr. Biol. 3, 327-332.

Posewitz, M. C., and Tempst, P. (1999). Immobilized gallium(III) affinity chromatography of phosphopeptides. Anal. Chem. 71, 2883-2892.

Qin, G., Meng, X., Wang, Q., and Tian, S. (2009). Oxidative damage of mitochondrial proteins contributes to fruit senescence: a redox proteomics analysis. J. Proteome Res. 8, 2449-2462.

Randall, D. D., Williams, M., and Rapp, B. J. (1981). Phosphorylationdephosphorylation of pyruvate dehydrogenase complex from pea leaf mitochondria. Arch. Biochem. Biophys. 207, 437-444.

Rao, R. S., and Møller, I. M. (2012). Large-scale analysis of phosphorylation site occupancy in eukaryotic proteins. Biochim. Biophys. Acta 1824, 405-412.

Rasmusson, A. G., Geisler, D. A., and Møller, I. M. (2008). The multiplicity of dehydrogenases in the electron transport chain of plant mitochondria. Mitochondrion 8 47-60.

Rasmusson, A. G., and Møller, I. M. (2011). "Mitochondrial electron transport and plant stress," in Plant Mitochondria, Chapter 14, ed F. Kempken (New York, NY: Springer), 357-381.

Rasmusson, A. G., Soole, K. L., and Elthon, T. E. (2004). Alternative $\mathrm{NAD}(\mathrm{P}) \mathrm{H}$ dehydrogenases of plant mitochondria. Annu. Rev. Plant Biol. 55, 23-39.

Reinders, J., Wagner, K., Zahedi, R. P., Stojanovski, D., Eyrich, B., van der Laan, M., et al. (2007). Profiling phosphoproteins of yeast 
mitochondria reveals a role of phosphorylation in assembly of the ATP synthase. Mol. Cell. Proteom. 6, 1896-1906.

Robson, C. A., and Vanlerberghe, G. C. (2002). Transgenic plant cells lacking mitochondrial alternative oxidase have increased susceptibility to mitochondria-dependent and -independent pathways of programmed cell death. Plant Physiol. 129, 1908-1920.

Rubin, P. M., and Randall, D. D. (1977). Regulation of plant pyruvate dehydrogenase complex by phosphorylation. Plant Physiol. 60, 34-39.

Schmidt, O., Harbauer, A. B., Rao, S., Eyrich, B., Zahedi, R. P., Stojanovski, D., et al. (2011). Regulation of mitochondrial protein import by cytosolic kinases. Cell 144, 227-239.

Schulenberg, B., Aggeler, R., Beechem, J. M., Capaldi, R. A., and Patton, W. F. (2003). Analysis of steady-state protein phosphorylation in mitochondria using a novel fluorescent phosphosensor dye. J. Biol. Chem. 278, 27251-27255.

Schuller, K. A., and Randall, D. D. (1990). Mechanism of pyruvate inhibition of plant pyruvate dehydrogenase kinase and synergism with ADP. Arch. Biochem. Biophys. 278, 211-216.

Sommarin, M., Petit, P. X., and Møller, I. M. (1990). Endogenous protein phosphorylation in purified plant mitochondria. Biochim. Biophys. Acta 1052, 195-203.

Stensballe, A., Andersen, S., and Jensen, O. N. (2001). Characterization of phosphoproteins from electrophoretic gels by nanoscale $\mathrm{Fe}(\mathrm{III})$ affinity chromatography with off-line mass spectrometry analysis. Proteomics 1, 207-222.
Struglics, A., Fredlund, K. M., Møller, I. M., and Allen, J. F. (1998). Two subunits of the F0F1-ATPase are phosphorylated in the inner mitochondrial membrane. Biochem. Biophys. Res. Commun. 243, 664-668.

Struglics, A., Fredlund, K. M., Rasmusson, A. G., and Møller, I. M. (1993). The presence of a short redox chain in the membrane of intact potato-tuber peroxisomes and the association of malate-dehydrogenase with the peroxisomal membrane. Physiol. Plant. 88, 19-28.

Sugiyama, N., Nakagami, H., Mochida, K., Daudi, A., Tomita, M., Shirasu, K., et al. (2008). Large-scale phosphorylation mapping reveals the extent of tyrosine phosphorylation in Arabidopsis. Mol. Syst. Biol. 4:193. doi: $10.1038 / \mathrm{msb} .2008 .32$

Takahashi, A., Kawasaki, T., Wong, H. L., Suharsono, U., Hirano, H., and Shimamoto, K. (2003). Hyperphosphorylation of a mitochondrial protein, prohibitin, is induced by calyculin $\mathrm{A}$ in a rice lesion-mimic mutant cdr1. Plant Physiol. 132, 1861-1869.

Tan, Y. F., Millar, A. H., and Taylor, N. L. (2012). Components of mitochondrial oxidative phosphorylation vary in abundance following exposure to cold and chemical stresses. J. Proteom. Res. 11, 3860-3879.

Taylor, N. L., Heazlewood, J. L., and Millar, A. H. (2011). The Arabidopsis thaliana 2-D gel mitochondrial proteome: refining the value of reference maps for assessing protein abundance, contaminants and post-translational modifications. Proteomics 11, 1720-1733.
Thelen, J. J., Miernyk, J. A., and Randall, D. D. (1998a). Partial purification and characterization of the maize mitochondrial pyruvate dehydrogenase complex. Plant Physiol. 116, 1443-1450.

Thelen, J. J., Muszynski, M. G., Miernyk, J. A., and Randall, D. D. (1998b). Molecular analysis of two pyruvate dehydrogenase kinases from maize. J. Biol. Chem. 273, 26618-26623.

Thelen, J. J., Miernyk, J. A., and Randall, D. D. (2000). Pyruvate dehydrogenase kinase from Arabidopsis thaliana: a protein histidine kinase that phosphorylates serine residues. Biochem. J. 349, 195-201.

Tolleter, D., Jaquinod, M., Mangavel, C., Passirani, C., Saulnier, P., Manon, S., et al. (2007). Structure and function of a mitochondrial late embryogenesis abundant protein are revealed by desiccation. Plant Cell 19, 1580-1589.

Tovar-Mendez, A., Miernyk, J. A., and Randall, D. D. (2003). Regulation of pyruvate dehydrogenase complex activity in plant cells. Eur. J. Biochem. 270, 1043-1049.

Vanlerberghe, G. C., and McIntosh, L. (1997). Alternative oxidase: from gene to function. Annu. Rev. Plant Phys. 48, 703-734.

Vercesi, A. E., Martins, I. S., Silva, M. A. P., Leite, H. M. F., Cuccovia, I. M., and Chaimovich, H. (1995). Pumping plants. Nature 375, 24-24.

Vidal, V., Ranty, B., Dillenschneider, M., Charpenteau, M., and Ranjeva, R. (1993). Molecular characterization of a $70 \mathrm{kDa}$ heat-shock protein of bean mitochondria. Plant J. 3 143-150.
Wold, F. (1981). In vivo chemical modification of proteins (posttranslational modification). Annu. Rev. Biochem. 50, 783-814.

Yao, Q., Bollinger, C., Gao, J., Xu, D., and Thelen, J. J. (2012) P(3)DB: an integrated database for plant protein phosphorylation. Front. Plant Sci. 3:206. doi: 10.3389/fpls.2012.00206

Zhu, Y., Lu, J. F., Wang, J., Chen, F., Leng, F. F., and Li, H. Y. (2011). Regulation of thermogenesis in plants: the interaction of alternative oxidase and plant uncoupling mitochondrial protein. J. Integr. Plant Biol. 53, 7-13.

Conflict of Interest Statement: The authors declare that the research was conducted in the absence of any commercial or financial relationships that could be construed as a potential conflict of interest.

Received: 03 December 2012; paper pending published: 03 January 2013; accepted: 26 February 2013; published online: 13 March 2013.

Citation: Havelund JF, Thelen JJ and Møller IM (2013) Biochemistry, proteomics, and phosphoproteomics of plant mitochondria from non-photosynthetic cells. Front. Plant Sci. 4:51. doi: 10.3389/ fpls.2013.00051

This article was submitted to Frontiers in Plant Proteomics, a specialty of Frontiers in Plant Science.

Copyright (c) 2013 Havelund, Thelen and Møller. This is an open-access article distributed under the terms of the Creative Commons Attribution License, which permits use, distribution and reproduction in other forums, provided the original authors and source are credited and subject to any copyright notices concerning any third-party graphics etc. 